\title{
Flow analysis and validation of numerical modelling for a thin walled high pressure die casting using SPH
}

\author{
Paul W. Cleary • Gary Savage • Joseph Ha • \\ Mahesh Prakash
}

Received: 22 December 2013 / Revised: 1 April 2014 / Accepted: 12 May 2014 / Published online: 28 May 2014

(C) OWZ 2014

\begin{abstract}
High pressure die casting (HPDC) is an important process for high throughput manufacturing of complex shaped metallic components. The flow involves significant fragmentation and spray formation as the high pressure liquid jets into the die from the gate system. An important class of die cast components is one with large areas of thin walls. An example of this is the chassis of the laptop computer. Computational modelling provides an opportunity to both better understand the filling process and to optimize the runner, gates, flash overs and venting systems for the die. SPH has previously been found to be very suitable for predicting HPDC for bulkier automotive components. The modelling challenges arising from the very thin sections and the many flow paths in a laptop chassis require careful validation. A water analogue experiment is used to validate the predictions of the SPH model for this representative thin walled casting. SPH predictions are used to understand and characterise the filling process. Finally, comparison of flow lines visible in an etched finished casting with the high speed flow paths in the final filled SPH model show very strong agreement. Together these demonstrate that such an SPH model is able to capture substantial detail from both the water analogue system and the actual casting process and is very suitable for simulating these types of complex thin walled castings.
\end{abstract}

Keywords High pressure die casting - HPDC - Smoothed particle hydrodynamics - SPH · Validation - Thin walls · water analogue

P. W. Cleary $(\bowtie) \cdot$ J. Ha $\cdot$ M. Prakash

CSIRO Mathematics, Informatics and Statistics, Private Bag 33,

Clayton South, VIC 3168, Australia

e-mail: paul.cleary@csiro.au

G. Savage

CSIRO Materials Science and Engineering,

Clayton South, VIC 3168, Australia

\section{Introduction}

High pressure die casting (HPDC) is an important process in the manufacturing of high volume and low cost metal components for the automotive, household products and electronics industries. Liquid metal (generally aluminium, magnesium or zinc) is injected into the die at high speed (30$100 \mathrm{~m} / \mathrm{s}$ ) and under high pressure through complex gate and runner systems. The geometric complexity of the dies leads to strongly three dimensional fluid flow with significant free surface fragmentation and splashing. The order in which the various parts of the die fill and the positioning of the air vents are crucial to forming homogeneous cast components with minimal entrapped voids. This is influenced by the design of the gating system and the geometry of the die. Numerical simulation offers a powerful and cost effective way to study the effectiveness of different die designs and filling processes, ultimately leading to improvements in both product quality and process productivity, including more effective control of the die filling and die thermal performance.

A simulation technique that has proven to be very effective at modelling these HPDC flows is smoothed particle hydrodynamic (SPH). See Monaghan [21-23] for a review of the basic method and Cleary et al. [9] for a review of its use in industrial applications, such as die casting. SPH is a Lagrangian (grid-free) method for modelling heat and mass flows and is well suited to simulating the complex splashing free surface flows found here. In SPH, materials are approximated by particles that are free to move around rather than by fixed grids or meshes. The particles are moving interpolation points that carry with them physical properties, such as the mass of the fluid, its temperature, enthalpy, density and any other properties that are relevant. The inter-particle forces are calculated by smoothing the information from nearby particles in a way that ensures that the resultant particle motion is 
consistent with the motion of a corresponding real fluid, as determined by the Navier-Stokes equations.

SPH has the following advantages for modelling various die casting processes:

- Complex free surface and material interface behaviour, including fragmentation, can be modelled easily and naturally.

- The Lagrangian framework means that there is no nonlinear term in the momentum equation, thus the method handles momentum dominated flows very well.

- Ability to include thermal shrinkage.

- Ability to easily track microstructure and composition information.

- Ability to predict oxide formation and gas entrapment directly as part of the simulations.

Complicated physics such as multiple phases, realistic equations of state, compressibility, solidification, fracturing, porous media flow, electromagnetics and history dependence of material properties are also easier to implement than in Eulerian methods. SPH was introduced for modelling high pressure die casting by Cleary et al. [3]. Ha [14] applied this for the first time to modelling HPDC in three dimensions for a simple machine component. Cleary et al. [5,6] suggested improved methods of visualizing the 3D HPDC SPH simulations and also compared SPH simulations of gravity die casting with experimental and MAGMAsoft simulated results. In [16] the effect of Reynolds number and resolution of the SPH solution were analysed for the HPDC of a machine component. Cleary et al. [7,8] applied SPH to predicting HPDC in a range of automotive products. Cleary et al. [11] then applied SPH to a broader range of product types including house hold products (such as door handles cast from zinc) and housings for electronic components.

Validation has so far been mainly performed using water analogue experiments. Ha and Cleary [4] used existing published water analogue experiments in a simple quasi-2D configuration to show the advantages of SPH in capturing critical thin films and small scale flow features. Good quantitative agreement between SPH simulations and water analogue experiments was also obtained for gravity die casting in $\mathrm{Ha}$ et al. [15]. Cleary et al. [7,8] performed detailed comparison between SPH, MagmaSoft simulations and water analogue experiments for a relatively compact automotive component. This showed much better agreement for the SPH predictions. Cleary et al. [11] also explored the impact of a range of thermal operating conditions on the filling of a thin wall coaster using SPH. The simulations included heat transfer and solidification coupled to the fluid flow and were compared with experimental short shots showing good agreement.

Due to its ability to track history dependent properties SPH has also been used to evaluate and optimize oxide levels in
Al re-melt ingot casting by Prakash et al. [27] and during furnace tipping operations in Prakash et al. [28-30]. It has been used for metal forming operations such as forging by Bonet and Kulasegaram [1] and Cleary et al. [7,8,10]. Cleary [2] extended the SPH method to predict feeding, freezing and defect creation in low pressure die casting applications. More recently, Pineau and D'Amours [26] used SPH to predict skin inclusions in semi-solid metal castings. Vijaykumar [35] also used SPH to investigate its ability to model continuous casting of steel.

Other methods sometimes used for modelling casting include the finite element method (using FIDAP) by Schneider and Grun [34] who modelled flow and temperature during direct chill (DC) casting table filling. Hu et al. [18] used the finite difference based MAGMAsoft to design and optimize the runner and gating system for the die-casting of a thin walled magnesium telecommunication component. Pellika and Wendt [25] used the finite element based software ProCAST to demonstrate the advantages of numerical simulation in modeling the HPDC of a simple aluminium coaster with heat transfer and solidification. Helenius et al. [17] also used MAGMAsoft to predict the heat transfer and flow during the filling of a HPDC shot sleeve. Rai et al. [31] used ProCAST in combination with an artificial neural network model to predict the filling time, solidification time and porosity in a simple HPDC component by setting an optimality criterion on four industrially relevant process parameters. Kermanpur et al. [19] simulated the metal flow and solidification in a multi-cavity mould for automotive components using the finite volume code FLOW-3D. As described in this paper, conventional grid based methods find it difficult to generate good quality meshes for complex geometries when using the finite element method. Lee et al. [20] used the 3D finite element code DEFORM-3D to analyse internal void defects during open die forging of large ingots into wrought iron components.

Gunasegaram et al. [13] used the finite difference based MAGMAsoft in conjunction with the concept of design of experiments (DOE) to limit the number of simulations required for optimizing a given set of process parameters. For finite difference and finite volume methods, without careful special treatments, one typically encounters a "stair-step" representation of curved geometries which leads to artificial numerical resistance to flow and flow artifacts if the surfaces are not resolved at a very fine resolution.

Pang et al. [24] used a particle level set method to simulate two phase flows of cast filling process. This is the first attempt at numerically evaluating back pressure effects during die casting processes. However, the simulations were performed only for low pressure die casting applications for simple geometries.

The use of a grid free method such as SPH means that issues relating to the numerical representation of the geome- 
try are able to be avoided as well as the difficulties in creating high quality meshes for geometrically very complex components. The ability of SPH to naturally resolve the free surface shape without use of surface tracking methods such as the ones described in Rudman [33] and Rider and Kothe [32], is also strongly advantageous.

In this paper, the use of SPH is extended to modelling HPDC flow in castings with large areas of thin walls. The indicative example used is that of casting a laptop chassis. The flow is characterised by a central location of the shot sleeve, radial runners and divergent radial flow outwards through the thin walled base plate with orthogonal thin walled structures. This is a very challenging casting geometry with proportionally larger areas of sections that are relatively thinner than in previous studies. Since economic pressures and requirements for light weighting (in both automotive and mobile devices) are towards ever thinner walls, it is important that numerical simulation also be tested and validated for these types of casting geometries. So here we compare the detailed isothermal filling pattern predicted by SPH to water analogue experiment. We also compare the predicted flow lines during feeding to the frozen flow lines that are visible in etched examples of the laptop casting. Both forms of experimental comparison demonstrate that the prediction of thin walled HPDC by SPH has good accuracy.

\section{The SPH methodology}

\subsection{SPH function and gradient estimation}

Here, we provide only a brief summary of the method. For more comprehensive details on the method see Monaghan [21,22] and Cleary et al. [9]. The interpolated value of a function $\mathrm{A}$ at any position $\mathbf{r}$ can be expressed using $\mathrm{SPH}$ smoothing as:

$A(\mathbf{r})=\sum_{b} m_{b} \frac{A_{b}}{\rho_{b}} W\left(\mathbf{r}-\mathbf{r}_{b}, h\right)$

where $m_{\mathrm{b}}$ and $\mathbf{r}_{\mathrm{b}}$ are the mass and density of particle $\mathrm{b}$ and the sum is over all particles b within a radius $2 \mathrm{~h}$ of $\mathbf{r}$. Here $W(\mathbf{r}, \mathrm{h})$ is a $\mathrm{C}^{2}$ spline based interpolation or smoothing kernel with radius $2 \mathrm{~h}$, that approximates the shape of a Gaussian function but has compact support. The gradient of the function $\mathrm{A}$ is given by differentiating the interpolation Eq. (1) to give:

$\nabla A(\mathbf{r})=\sum_{b} m_{b} \frac{A_{b}}{\rho_{b}} \nabla W\left(\mathbf{r}-\mathbf{r}_{b}, h\right)$

Using these interpolation formula and suitable finite difference approximations for second order derivatives, one is able to convert parabolic partial differential equations into ordi- nary differential equations for the motion of the particles and the rates of change of their properties.

\subsection{Continuity equation}

From Monaghan [21], our preferred form of the SPH continuity equation is:

$\frac{\mathrm{d} \rho_{a}}{\mathrm{~d} t}=\sum_{b} m_{b}\left(\mathbf{v}_{a}-\mathbf{v}_{b}\right) \nabla W_{a b}$

where $\mathbf{r}_{\mathrm{a}}$ is the density of particle $a$ with velocity $\mathbf{v}_{\mathrm{a}}$ and $m_{b}$ is the mass of particle $b$. We denote the position vector from particle $b$ to particle a by $\mathbf{r}_{\mathrm{ab}}=\mathbf{r}_{\mathrm{a}}-\mathbf{r}_{\mathrm{b}}$ and let $W_{\mathrm{ab}}=W\left(\mathbf{r}_{\mathrm{ab}}, \mathrm{h}\right)$ be the interpolation kernel with smoothing length h evaluated for the distance $\left|\mathbf{r}_{\mathrm{ab}}\right|$. This form of the continuity equation is Galilean invariant (since the positions and velocities appear only as differences), has good numerical conservation properties and is not affected by free surfaces or density discontinuities. The use of this form of the continuity equation is very important for predicting free surface flows such as those occurring in die casting [4].

\subsection{Equation of state}

The SPH method used here is compressible and is used near the incompressible limit by choosing a sound speed that is much larger than the velocity scales in the flow. The equation of state, giving the relationship between particle density and fluid pressure, is:

$P=P_{0}\left[\left(\frac{\rho}{\rho_{0}}\right)^{\gamma}-1\right]$

where $P_{0}$ is the magnitude of the pressure and $\rho_{0}$ is the reference density. For water or liquid metals we use $\gamma=7$. The pressure scale factor $P_{0}$ is given by:

$$
\frac{\gamma P_{0}}{\rho_{0}}=100 \mathrm{~V}^{2}=c_{s}^{2}
$$

where $V$ is the characteristic or maximum fluid velocity. This ensures that the density variation is less than $1 \%$ and the flow can be regarded as incompressible.

\subsection{Momentum equation}

The SPH momentum equation used here, using the pressure from Eq. (4), to predict the particle motion:

$$
\begin{aligned}
\frac{\mathrm{d} \mathbf{v}_{\mathbf{a}}}{\mathrm{dt}}= & \mathbf{g}-\sum_{b} m_{b} \\
& \times\left[\left(\frac{P_{b}}{\rho_{b}^{2}}+\frac{P_{a}}{\rho_{a}^{2}}\right)-\frac{\xi}{\rho_{a} \rho_{b}} \frac{4 \mu_{a} \mu_{b}}{\left(\mu_{a}+\mu_{b}\right)} \frac{\mathbf{v}_{a b} \cdot \mathbf{r}_{a b}}{\mathbf{r}_{a b}^{2}+\eta^{2}}\right] \\
& \nabla_{a} W_{a b}
\end{aligned}
$$


where $P_{\mathrm{a}}$ and $\mu_{\mathrm{a}}$ are pressure and viscosity of particle a and $\mathbf{v}_{\mathrm{ab}}=\mathbf{v}_{\mathrm{a}}-\mathbf{v}_{\mathrm{b}}$. Here $\mathrm{h}$ is a small parameter used to smooth out the singularity at $\mathbf{r}_{\mathrm{ab}}=0$ and $\mathbf{g}$ is the gravity vector. The first two terms involving the pressure correspond to the pressure gradient term of the Navier-Stokes equation. The next term involving viscosities is the Newtonian viscous stress term. This form ensures that stress is automatically continuous across material interfaces and allows the viscosity to be variable or discontinuous. Multiple materials with viscosities varying by up to five orders of magnitude can be accurately simulated.

The nature of the interaction between these SPH equations can be understood by considering two particles. As they approach each other, their relative velocity is negative so that there is a positive contribution to $\mathrm{d} \rho_{\mathrm{a}} / \mathrm{dt}$ causing $\rho_{a}$ to rise, leading to a positive pressure that pushes the particles apart again. As two particles move apart their densities decrease creating a negative pressure that pulls the particles back towards each other. This interplay of velocity and density/pressure ensures that the particles remain 'on average' equally spaced and that the density is close to uniform so that the fluid is close to incompressible.

\section{Water analogue experimental apparatus}

Water analogue methods for the validation of high pressure casting simulation models have been widely adopted for many years with one of the first such attempts made by Davies et al. [12]. This is based on the similarity of the kinematic viscosity of water to that of liquid metals such as aluminium and magnesium, so that the Reynolds number for the water and metal systems are comparable leading to similarity in the flow behaviour.

A perspex mould was manufactured from 3D CAD data derived from an actual casting of the laptop cover, complete with sprue, runners, gates and overflows as shown in Fig. 1. The casting is $277 \mathrm{~mm}$ wide by $216 \mathrm{~mm}$ high by 20 deep. The main wall section thickness for the chassis was $1.6 \mathrm{~mm}$. The perimeter of the gate is $175 \mathrm{~mm}$ and the gate thickness is $0.9 \mathrm{~mm}$. The weight of the casting and overflows was 223 $\mathrm{g}$ and the gate area was $156 \mathrm{~mm}^{2}$. The sprue diameter at the visible top of the finished casting is around $30 \mathrm{~mm}$.

The water analogue experimental setup consisted of a pressurized accumulator, partially filled with coloured water at 6 bar pressure, which was connected to the perspex mould through a valve. The perspex mould was backlit through a diffuser and filling was captured using a Kodak SR1000 Motion Corder Analyser high speed digital camera. This configuration was chosen to provide a constant pressure water delivery system and a cavity fill time of approximately $37 \mathrm{~ms}$. The cavity fill time was chosen to provide good resolution of the early stages of cavity filling, based on the maximum allowable camera frame rate of 1,000 fps.

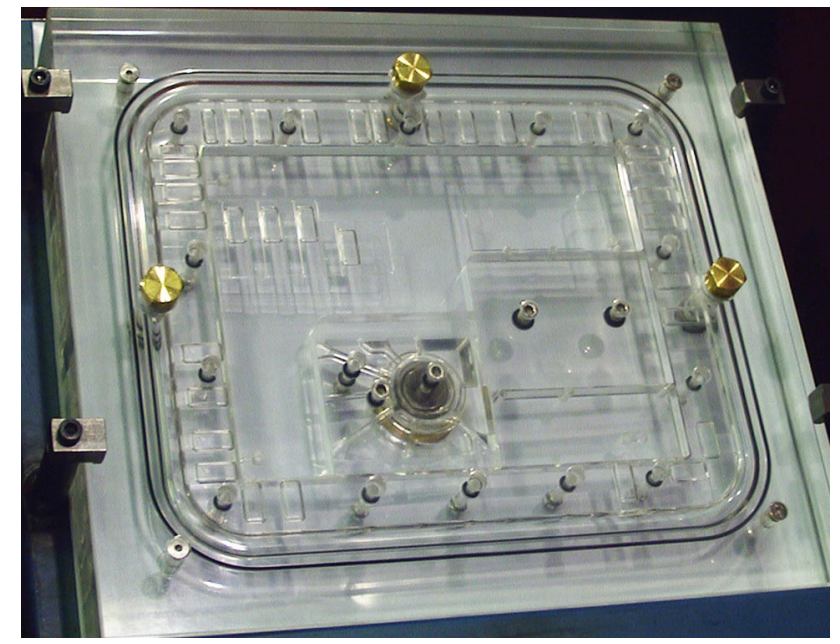

Fig. 1 Perspex model of the laptop cover used for water analogue flow experiments

\section{Modelling assumptions and setup}

The simulation was set up to exactly match the water analogue experiment with the geometry specified from the same CAD model used for making the perspex mould. The basic fluid properties used were density $=1,000 \mathrm{~kg} / \mathrm{m}^{3}$ and viscosity $=0.01 \mathrm{~Pa}$ s. This viscosity represents an estimate of the turbulent viscosity which is estimated to be around ten times higher than the kinematic viscosity of water. This viscosity level was found suitable in earlier validation work by Ha et al. [15] and Cleary et al. [7,8] leading to very good matches to the water analogue flow patterns. Previous testing has also shown low sensitivity of the flow predictions to the specific choice of viscosity in this range.

Since the water analogue flow rate was pressure controlled it varied moderately throughout the shot. We therefore used an estimated peak fluid speed in the shot sleeve of $3 \mathrm{~m} / \mathrm{s}$ which leads to maximum fluid speeds through the gate of around $33 \mathrm{~m} / \mathrm{s}$ to match the average experimental flow rate. Sensitivity of the predicted flow to the injection speed was also tested to estimate the error arising from this assumption. The choice of injection speed does not appear to affect the nature of the flow pattern but simply linearly scales the time for filling the die. These simulations used a resolution of $0.64 \mathrm{~mm}$ giving a maximum of 1.32 million SPH fluid particles when the die was completely filled. This would take around 1.5 days of cpu time on a current single core Intel cpu.

\section{The laptop configuration and nomenclature for description}

Figure 2 shows a photograph of the casting when viewed from below. This is the orientation/view used in the water 


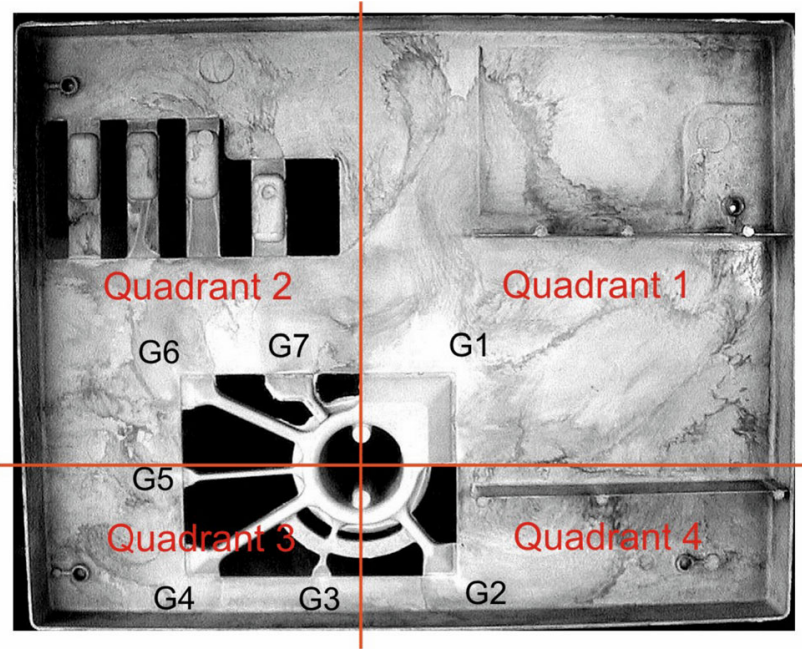

Fig. 2 Photograph of the casting viewed from below. Nomenclature for gates and quadrants is shown. The casting is $277 \mathrm{~mm}$ wide by 216 $\mathrm{mm}$ high by 20 deep. The perimeter of the gate is $175 \mathrm{~mm}$ and the gate thickness is $0.9 \mathrm{~mm}$

analogue experiments and later in Fig. 4 when comparing the SPH simulations to the experiment. In order to facilitate description of the filling process some nomenclatures is used to label the gates and the quadrants. These are shown in Fig. 2. The gates are numbered G1-G7 moving clockwise around the shot sleeve/runner system and starting with the big fan like gate in quadrant 1 . The quadrants are also numbered counter-clockwise.

\section{Filling of the laptop cover}

Figure 3 shows snapshots of the filling of the die from two views. The left view is from above (the direction of injection of the fluid) and the right view is from below. Both views are slightly angled so that flow in the vertical walls can be seen. The colour indicates fluid speed with dark blue being very slow and red being the highest speeds in the jets around the outside of the gates (around $32 \mathrm{~m} / \mathrm{s}$ ). The time in this simulation is measured from the start of the simulation when the fluid is created in the middle of the shot sleeve. The flow is rapid and complex, but space limitations prevent us including all the frames that are included in the description below. On average we include in Fig. 3 roughly every second time that is used in the analysis below.

At $4 \mathrm{~ms}$, the fluid has traveled down the shot sleeve and has just reached the runners. The fluid then flows through each of the runners and by $6 \mathrm{~ms}$ has entered the die. Since fluid leaves the runner under pressure and is entering a low pressure space, it diverges to form a thin diverging jet. A diverging jet must fragment in order to conserve volume and so fragmentation is visible at the lead- ing edges of the jets. By $8 \mathrm{~ms}$, the fluid has reached the lower edge of the die and the rectangular cutouts in quadrant 2

At the leading edge of the jets (particularly the large 90 degree fan shaped jet from G1) there is an accumulation of fluid that forms a "halo" of fluid moving more slowly than the fluid now jetting from the gates. This results from the incomplete pressurization of the runner system as the early fluid moves more slowly through the gates. The leading edge fluid at $6 \mathrm{~ms}$ is produced by the jet in the shot sleeve impacting the base of the die and spraying outwards at around $18 \mathrm{~m} / \mathrm{s}$. The remaining volume of the runners fills by back flow. When they are filled the fluid pressure in the runners rises rapidly and the fluid speed though the gate accelerates rapidly to around $32 \mathrm{~m} / \mathrm{s}$. This faster moving later entering fluid catches up with the slower moving early fluid to form a thicker band of fluid at the leading edge. This occurs because the air has been neglected and the simulation is equivalent to using an evacuated die. When air is present the pressurization of the runners occurs very early on so that the amount of slow moving material is very small. The existence of this larger band of fluid is the only known consequence (in terms of details of the flow pattern) for neglecting the air in the die.

By $10 \mathrm{~ms}$, most of the leading edge fluid has reached the edges of the die and fluid is seen discharging from most of the vents on the left and right sides of the die. Fluid is now flowing through the channels bridging the cutout in the upper part of the die (quadrant 2). The overall flow pattern is now well established with key features:

- The fluid flowing from the 90 degree fan-like gate G1 is not uniformly distributed in quadrant 1 , but is concentrated in two slowly diverging bands on the sides of the fan. There is a large central wedge shaped region into which little fluid is injected leading to a broad empty region in the opposite corner of the die.

- The jets only fill a small fraction of the volume. Much of the die to either side of the jets is filled by back flow from the outer edges of the die.

- Significant outflow through the vents is experienced, particularly through the vents that are directly opposite the inflow gates. This would suggest that such vent locations increase the fill time and may lead to more scrap being created in the vents than may be absolutely necessary.

- The amount of fluid injected into the different parts of the die does not appear to be well balanced. There is more fluid injected through the four gates on the cutout side of the die (G3-G7) than would appear necessary given the much smaller volume of die to fill in quadrants 2 and 3 . Conversely, there is not sufficient fluid injected through gate G1 so that this side of the die fills later than the other. 
Time $=6 \mathrm{~ms}$
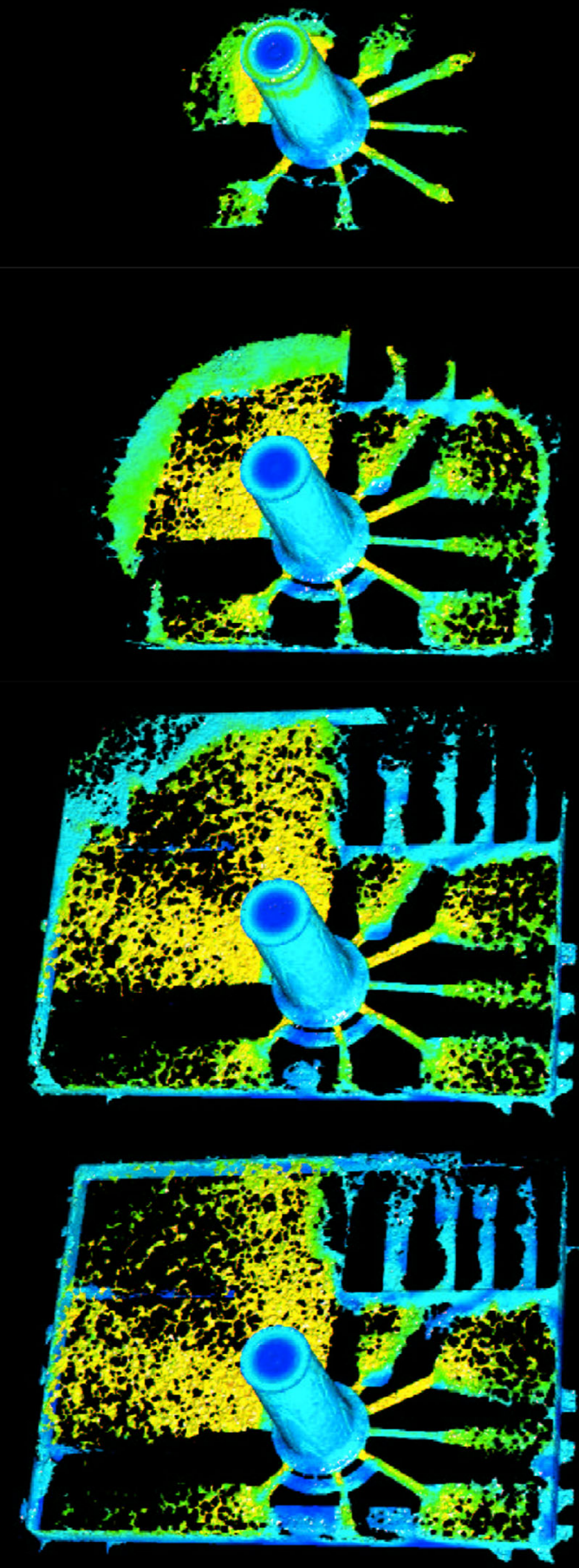

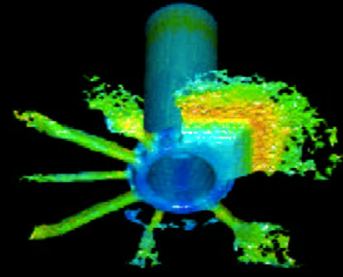

Time $=8 \mathrm{~ms}$

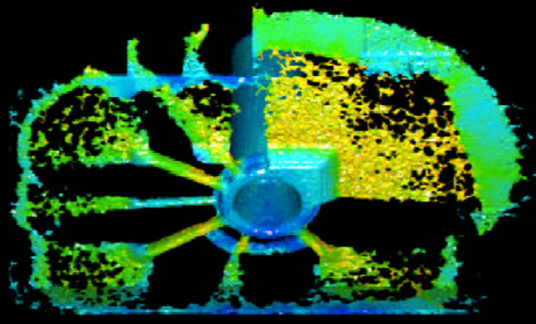

Time $=10 \mathrm{~ms}$

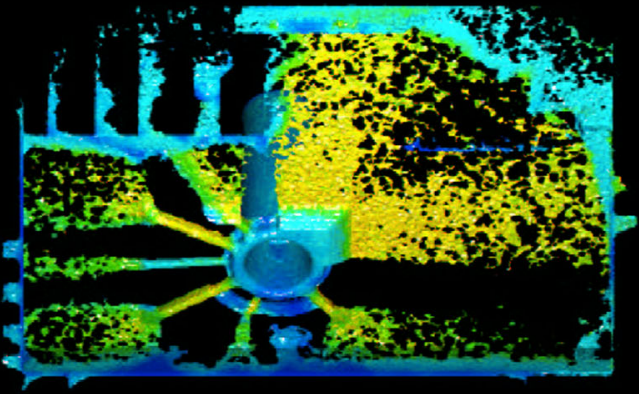

Time $=12 \mathrm{~ms}$

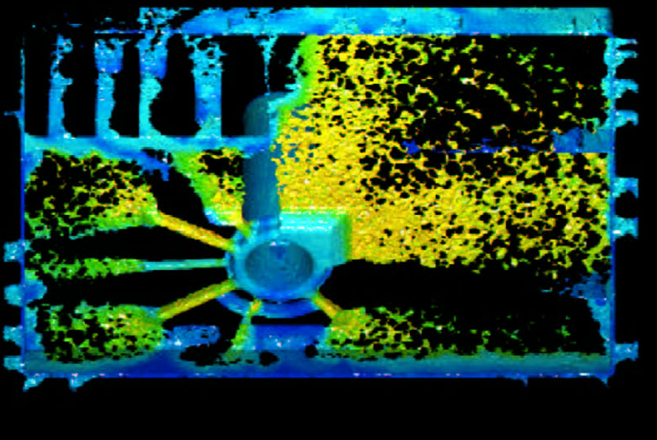

Fig. $3 \mathrm{SPH}$ predictions of the filling process with fluid is coloured by velocity (blue is slow, green is $16 \mathrm{~m} / \mathrm{s}$ and red is $33 \mathrm{~m} / \mathrm{s}$ ). (Left column) die is viewed from above and (right) view from below. Color figure online 


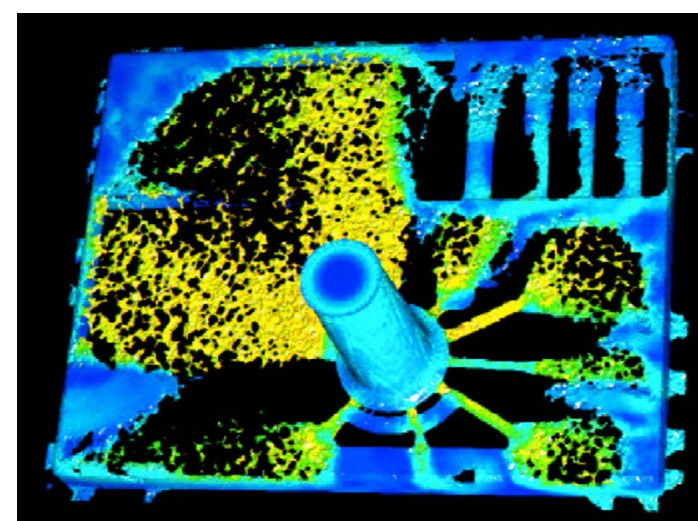

Time $=15 \mathrm{~ms}$

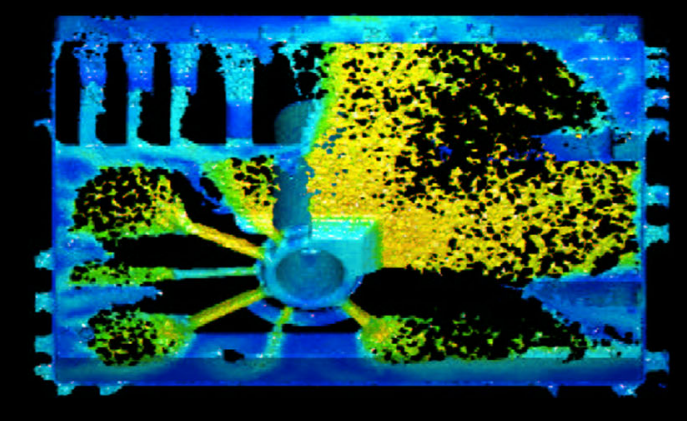

Time $=23 \mathrm{~ms}$
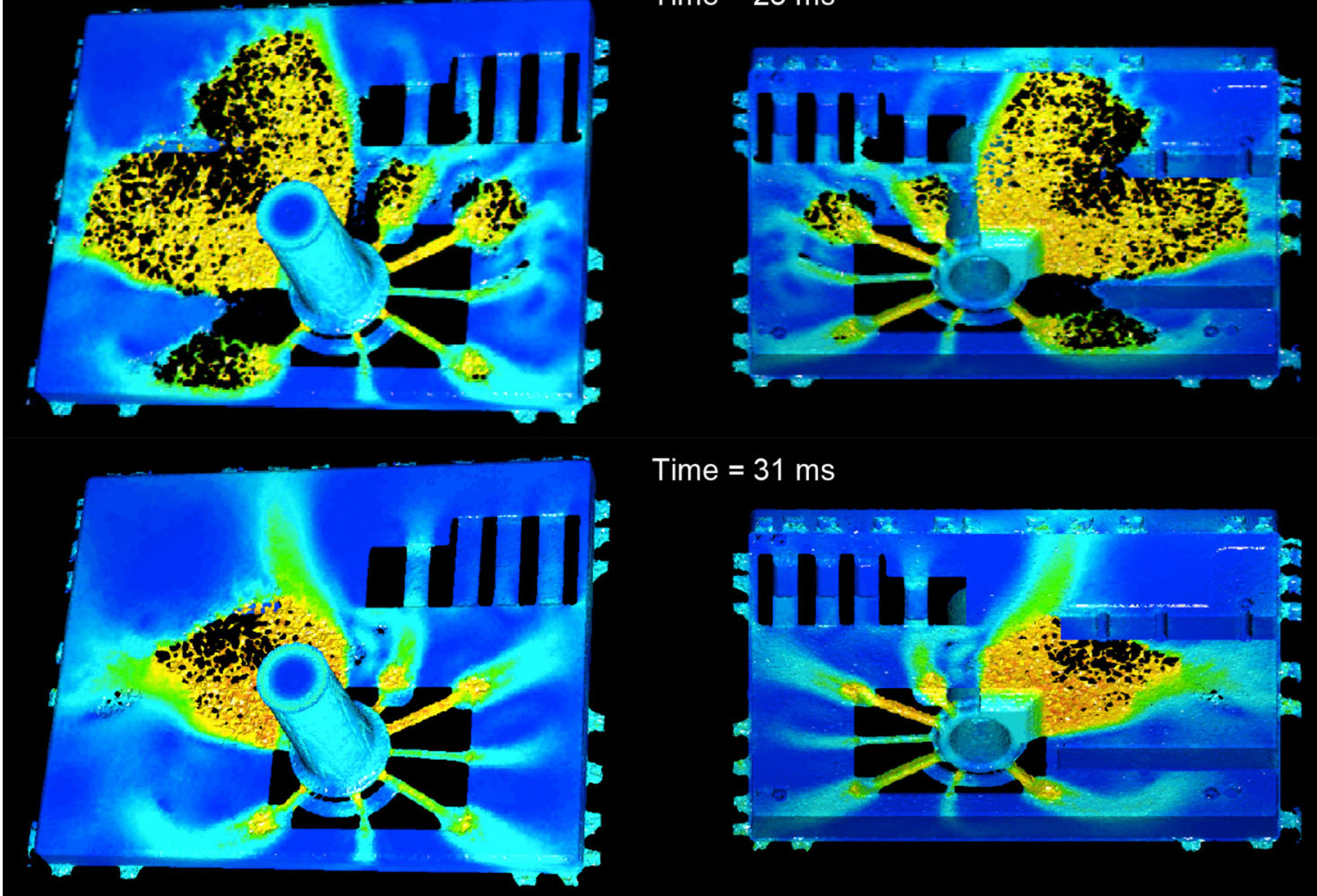

Time $=31 \mathrm{~ms}$

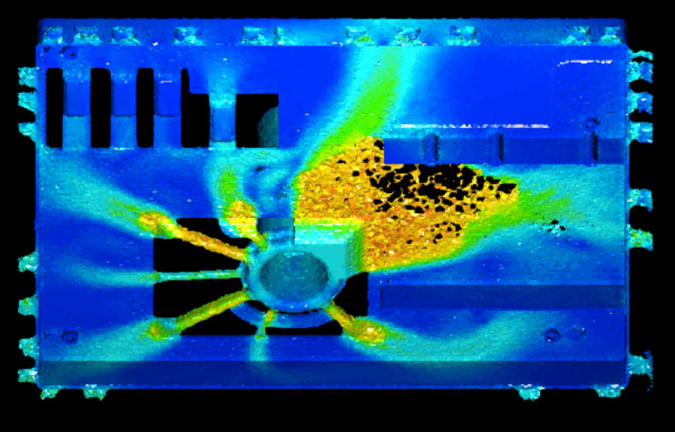

Time $=37 \mathrm{~ms}$
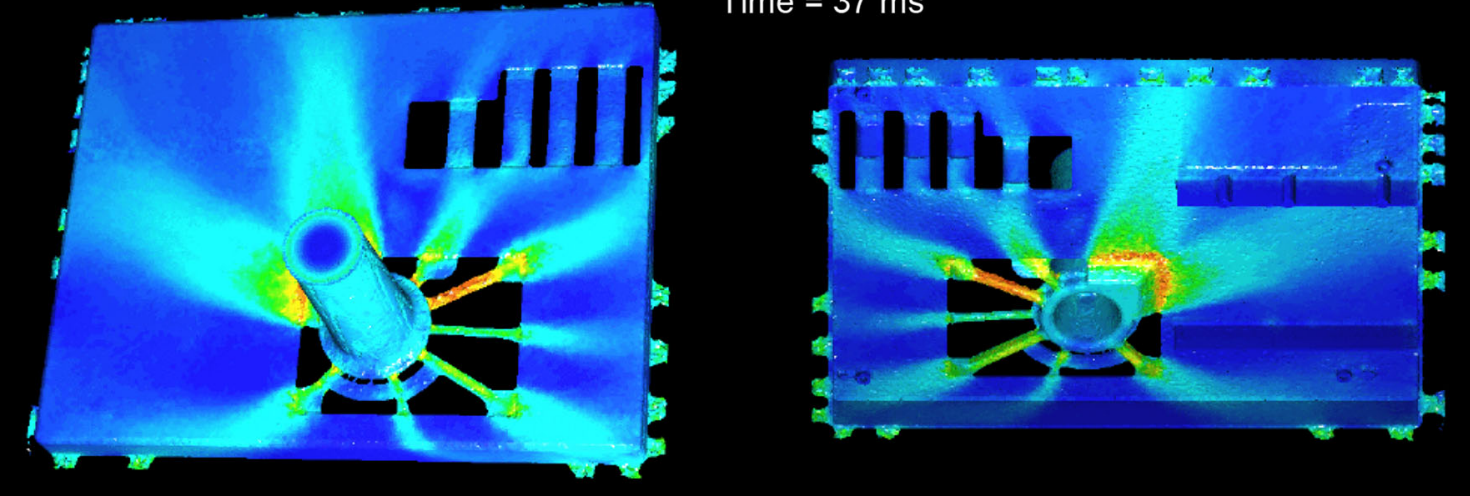

Fig. 3 continued 
A more balanced injection might reduce the fill time and the scrap produced by the flow into these vents. There is clearly insufficient fluid flow into quadrant 4 with the one thin runner struggling to get enough fluid into the quite large region beyond gate $\mathrm{G} 2$.

- In between the two gates at the bottom of the die (G2 and G3) a back filling jet can be seen starting to fill the region between the two outgoing jets coming from the gates.

- A layer of slow moving fluid has started to build up below the cutout in quadrant 2 of the die. These gaps in the casting and the thinner section thickness mean that flow up into the corner of the die in quadrant 2 is restricted. So fluid arrives at the bottom of the cutout faster than it can flow across the bridges or around and results in a buildup.

- There is a wake like flow from the bottom left corner of the cutout (in the view from above). This occurs because the jet from the gate G1 diverges upon entering the cavity due to the pressure applied in the runner. The edge of the jet impinges on the corner of the cutout and produces a turbulent, unsteady wake. This is augmented by some flow of fluid from the collection of fluid accumulating below the cutout. This effect is unlikely to be beneficial because it is likely to increase turbulence of void formation. This would be reduced if the shot sleeve were moved slightly so that the edge of the jet touches the edge of cutout much closer to edge of the die or avoids contact altogether.

At $12 \mathrm{~ms}$ the basic flow is similar with back filling between G2 and G3 almost completely filling the region between the jets. A similar back filling jet has started to fill the void between the two jets from gates G3 and G4 adjacent to the first pair. At this stage most of the vertical walls of the laptop cover have been filled, aside from the corner that is protected by the cutout. Fluid is now also starting to accumulate in the vertical supports on the side of the cover (right side when viewed from below).

At $13.5 \mathrm{~ms}$ fluid has started to accumulate on the side of the die below the cutout (quadrants 2 and 3) and back filling jets are now forming between the jets from gates G4-G7. The first back filling jet at the bottom of the die (between G2 and G3) has filled the void between the gates entirely. The second back filling region (between G3 and G4) has almost filled, except for a void trapped by the re-circulating fluid. At $15 \mathrm{~ms}$, the entire perimeter of the laptop cover is now filled. The void in the backfilled region between G3 and G4 continues to survive as the fluid continues to swirl around it. Significant back filling is now evident in the upper corner of quadrant 1 opposite the fan gate G1 and from the wall at the top of quadrant 4.
By $19 \mathrm{~ms}$, much of the die is filed. The narrow jets from the lower gates have filled the lower section of the die entirely and the jets on the side below the cutout (G4G6) have almost filled that side of the die. The region beyond the cutout in quadrant 2 is almost filled and the back filling across the top of quadrant 4 has more than half filled the large void in the lower left side of the die (when viewed from above). This back filling is very slow and the fluid at the leading edge has been resident in the die a long time. For a liquid metal, it would be relatively cold and one can envisage difficulties in getting this back filling jet to travel all the way to the die edge between the gates.

By $23 \mathrm{~ms}$, the voids around the jets from gates G4 and G5 have filled completely and the back flow between the jets from gates G6 and G7 has filled a large fraction of the voidage there. The backflow in quadrant 4 has filled around $3 / 4$ of the void area in this region and the two support ribs underneath the cover in quadrants 1 and 4 are substantially filled. All the voids behind the cut outs in quadrant 2 have finally filled and only two small voids remain in the bridges over the cut out.

The filling continues in a similar way slowly filling more of the component. The back filling process is extremely slow because of the short circuiting of the flow from the runner system out through the many vents. At $28 \mathrm{~ms}$, the laptop cover is around $90 \%$ full. The back flow in quadrant 4 has reached the inner edge of the die between gates G1 and G2 and has spread sideways leaving only some broken voids directly around the start of the jet from gate G2. Similarly, the flow from G7 has almost completely filled the voids between G7 and G1. The support ribs underneath the cover have also completed filling by this time. The majority of the quadrant 1 is now filled by the flow from gate G1. The small void in front of gate G6 has just filled so that the regions in front of gates G3-G6 have all now been completely filled.

By $31 \mathrm{~ms}$, the small voids in front of gates $\mathrm{G} 2$ and G7 have been filled leaving only the moderate void in quadrant 1 in front of gate G1. This void now reaches only as far into Q1 as the support rib and about half way from the gate to the side of the laptop cover. The filling of all the other voids means that more of the fluid entering from the runners now flows into this void and so it fills at a faster rate. By 34.5 $\mathrm{ms}$, this void has shrunk to a small region directly in front of the gate. At around $36.5 \mathrm{~ms}$, the final void in front of gate G1 fills and the flow reaches an equilibrium with the flow in through the runners matching the flow out through the vents. The colouring of the fluid surface in the frame at $37 \mathrm{~ms}$ shows this equilibrium velocity distribution very clearly, with high speeds at the gates leading to clear preferential paths through the die as the fluid flows towards the vents. 


\section{Comparison with water analogue experiment}

The experiments used a video frame rate of 1000 frames per second and the frame exposure was also $1 \mathrm{~ms}$. During the millisecond image capture period the fluid moves a considerable distance and the fluid fronts become blurred. The simulation pictures are instantaneous and therefore show much more fine detail. For the purposes of comparison, one needs to imagine smearing the simulation pictures with the fluid fragments blending together. For agreement one needs to observe the fluid being in the same places and with an intensity of the grey in the photographs (which is dependent on the local fluid volume fraction) being consistent with the local fluid volume fraction in the simulation.

Figure 4 shows selected frames taken from the experimental video at the $1 \mathrm{~ms}$ frame rate on the left. The time shown here is the experimental time from the opening of the pressure valve. On the right are the simulation results at comparable times. The first simulation frame is matched to the first experimental frame to determine the offset in the different time origins. Overall there is very good agreement between the simulation and the experiment. We will not seek to make a detailed frame by frame comparison but will highlight key similarities and differences as we step through the filling process in time.

- The first of the two differences evident early in the filling are:

(1) The absence in the experiment of the thick layer of slower moving fluid that is visible at the leading edges of the jets in the simulations (see $37 \mathrm{~ms}$ for example).

(2) The jets in the experiment diverge more than for the simulation and there is an additional fluid flowing from the fan gate (G1) below the horizontal lower edge of the gate. These are thought to be the result of leakage around the gate insert in the die in the form a thin sheet of additional fluid. See for example the $42 \mathrm{~ms}$ frame. The horizontal jet (G5) seems to have a darker core that is consistent with the shape of the simulated jet. The lighter grey on either side may also be from a thin sheet of fluid leaking from under the gate insert.

- There is very good agreement on the shape and extent of the main jet from the fan gate G1. The simulations show two dense streams traveling from each of the sides of the square corner of the gate. These correspond closely to the dark parts of the jets in the experiment (see $40 \mathrm{~ms}$ for example). Inside these dense parts of the jet is a dilute flow of highly fragmented material. This corresponds well to the light grey part of the jet in the experiment. In both the experiment and the simulation little fluid passes through the square region adjacent to the upper right corner in quadrant 1 . It is perhaps not so obvious that such a gate shape should preferentially direct so much fluid up or sideways and so little up at 45 degrees to the corner of the die. However there is good agreement between the experiments and the simulations on this effect.

- The shedding of the turbulent wake structure from the inner most corner of the cutout where the fan gate jet impacts, occurs in both experiment and simulation at similar times and has similar size and structure.

- The overall filling process is shown to be by the jets from the gates impacting the outside of the die and backfilling into the voids in between in both simulation and experiment. These similarities are particularly clear in the lower left part of the die.

- There is a high degree of agreement in the back filling of the two regions at the bottom of the die (between G2 and G3 and between G3 and G4) both in the extent of the back filling, the shape of the remaining voids and the timing of the filling. In particular, note that a long lived void is trapped at the center of re-circulation (frame 44 $\mathrm{ms}$ ) in the second of these back filled regions between G3 and G4.

- The extent and timing of the flow of fluid through the bridges over the cutout in quadrant 2 match very well throughout the filling.

- The timing of fluid flowing out through the vents agrees well as does the shape of the fluid free surfaces in the vents.

- The rate of filling of the perimeter of the die is in good agreement.

- There is good agreement about the extent to which fluid injected into the die flows straight out through the many of the perimeter vents. The amount of flow through is significant. At the $47 \mathrm{~ms}$ stage, more than $20 \%$ of the injected fluid in the simulations has been discharged and some of the flows through the vents reach speeds of up to $10-15 \mathrm{~m} / \mathrm{s}$, which is a reasonable fraction of the gate speed of $32 \mathrm{~m} / \mathrm{s}$.

- There are noticeable differences in the filling of quadrant 4 resulting from the additional jetting of fluid below the supporting wall section (the lower of the two dark horizontal lines on the right of the water analogue experiment pictures) arising from leakage due to die distortion under the high pressure experienced in this part of the experiment.

- Although the left side of the die back fills in the experiments (as it does the simulation) there is little sign of back filling from the upper right corner (as there is in the simulation). In the experiment this region does not appear to fill but fluid continues to flow through the die and a large swirling air bubble appears to be trapped near the top right corner. 


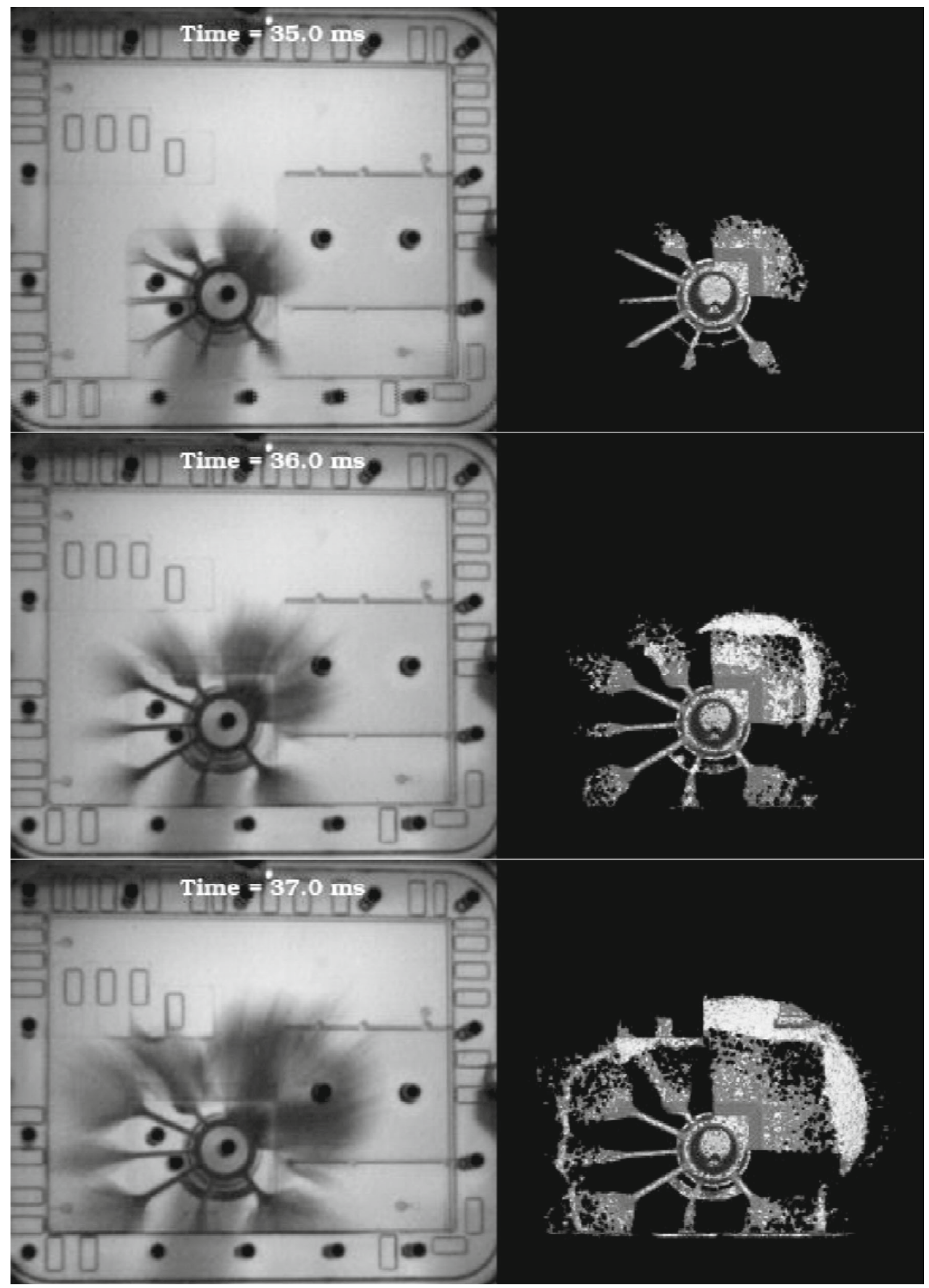

Fig. 4 Comparison between water-analogue (left) and SPH (right) results 


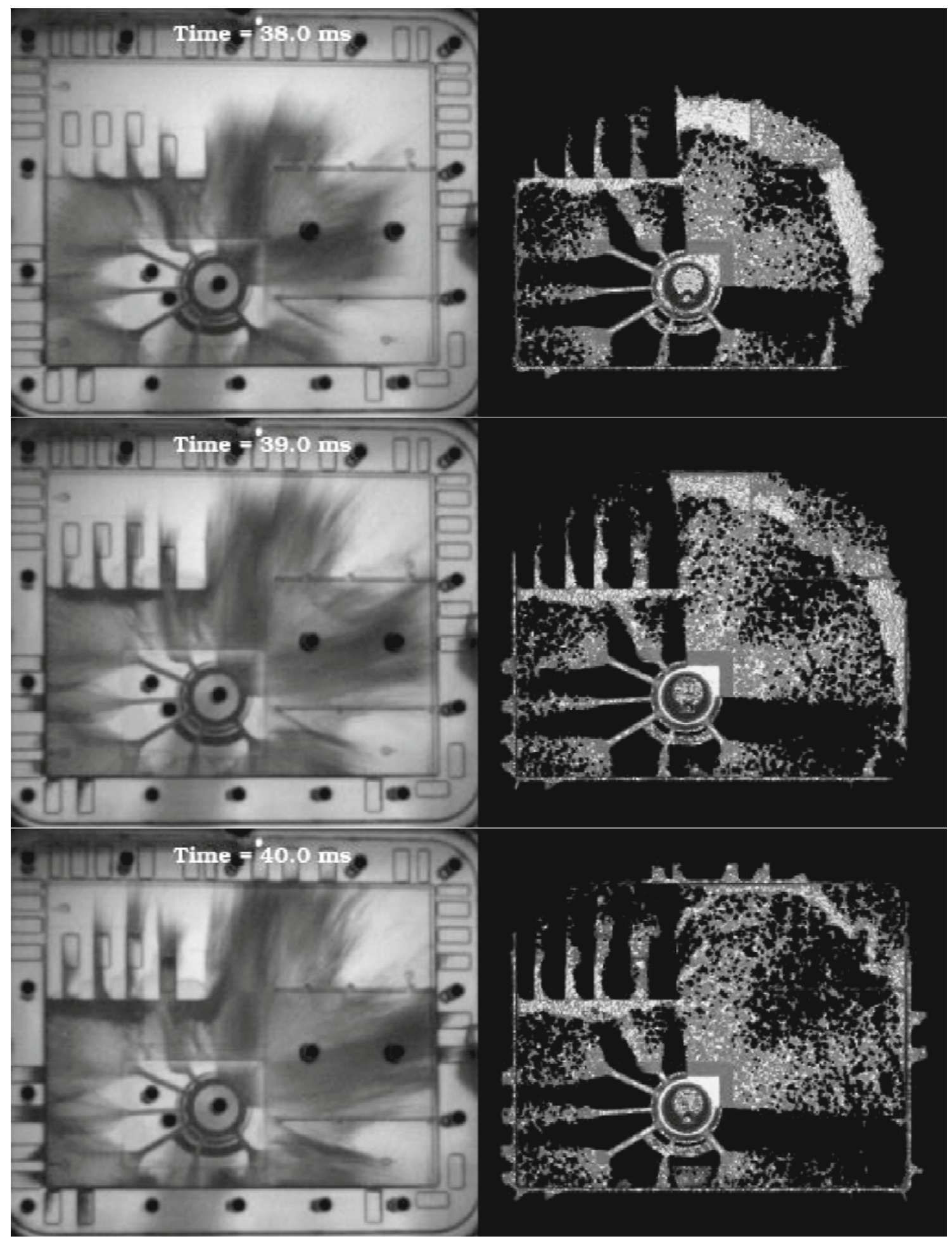

Fig. 4 continued 


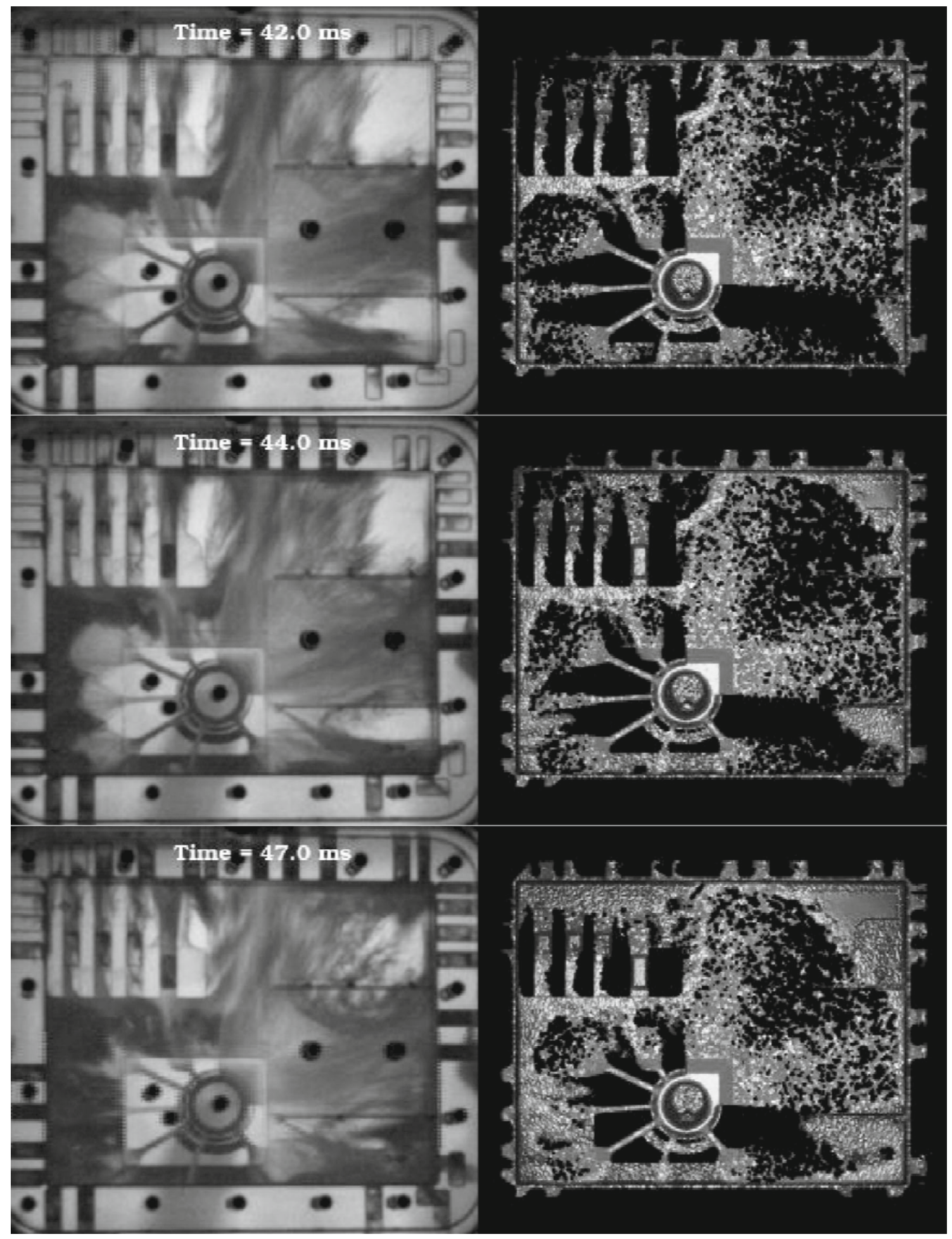

Fig. 4 continued 
Flow line consistent with the

fan jet being deflected from the corner of the rectangular

cutout

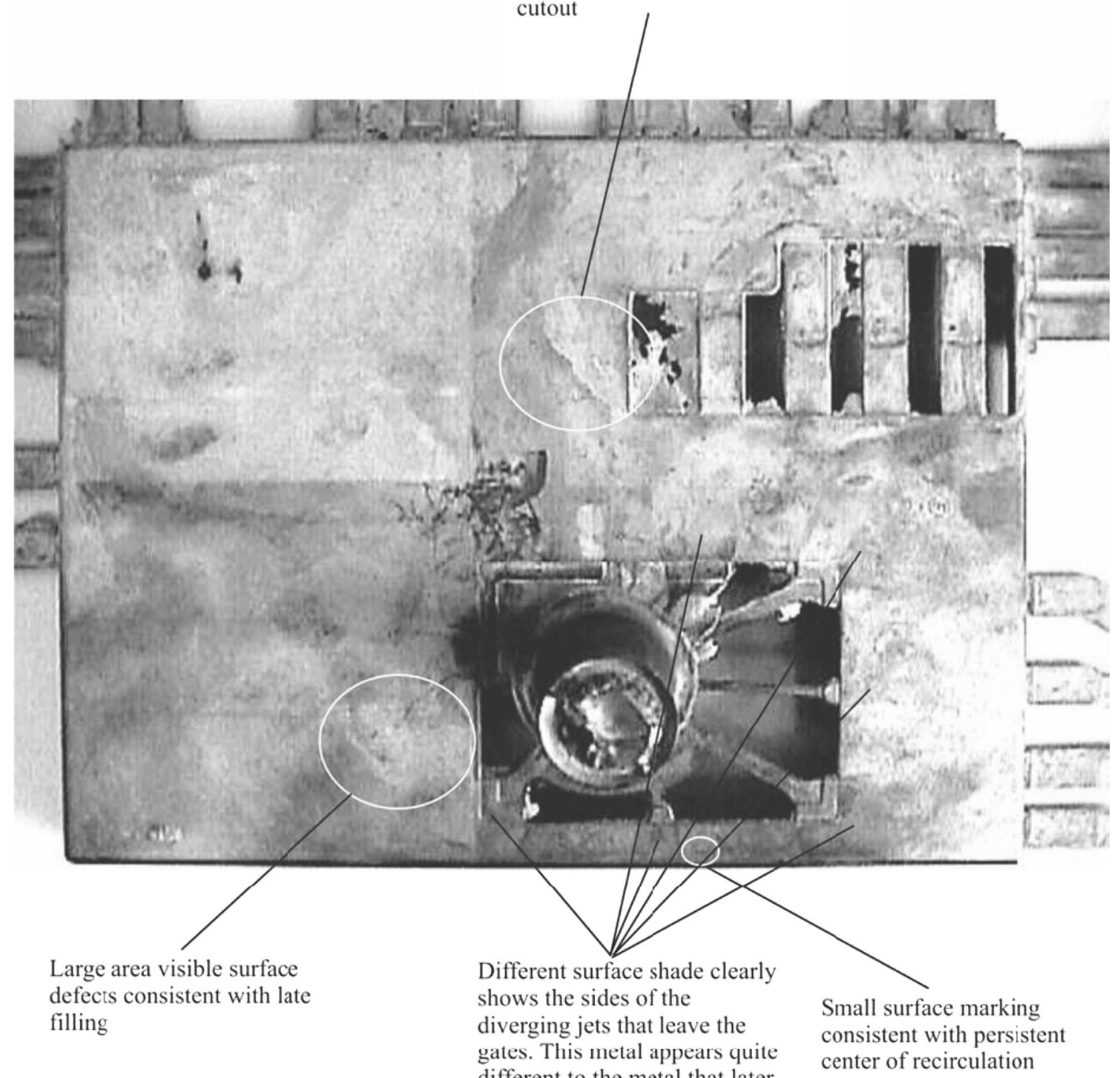

different to the metal that later

back fills into the die between

these jets

Fig. 5 Photograph of the casting of the laptop cover (from above) with flow related features indicated

\section{Comparison with surface features on the cast part}

It is also useful to compare the simulation predictions to the final cast component. Since solidification is heavily influenced by the flow and the flow history is frozen into the casting, we are able to deduce information about the flow from the microstructure observed in a final casting. This is facilitated by slightly etching the casting to reveal the differing microstructure. The feeding lines, produced by flow through of new metal after the die is filled but before flow from the shot sleeve has finished, are clearly identifiable. These are regions of smooth metal of uniform shade that form smooth narrow paths from the gates to the vents.

Several observable features on the casting shown in Fig. 5 correspond to parts of the predicted flow:

- The feeding lines emanating from the gates (as marked by the frozen in flow lines) are clearly visible in the surface finish of the cast part. The shape, size and positioning of these jets are in very close agreement with the SPH predictions of the jets (as shown by the non-blue material in the filled last frame of Fig. 3). In between the metal 
has very different colouring and texture which is highly consistent with the prediction that this is filled later by back flows from the perimeter.

- The location of the long lived vortex between gates G3 and G4 corresponds to the position of a dark more uneven patch on the cast part. This flow structure is able to trap gas which affects the final microstructure making it prone to porosity there. This matches the observed poor surface quality in the same region of the etched casting.

- There is a clearly visible swirling flow line arising from the inner most corner of the cutout. This is consistent with the turbulent wake shed by the corner in both the simulation and the experiment.

- In quadrant 4 , the simulation predicts that filling will occur by a slow back filling from the side wall and that this material will be old and therefore expected to be quite cold and semi-solid. It is no surprise that the last part of this region (nearest the gate) has clear signs of an uneven and partially filled surface. This area would seem to be prone to only partial filling and a strong candidate for consistent porosity generation.

\section{Conclusions}

SPH has been used to predict the filling of a representative very thin walled complex shaped casting - that of a laptop cover. This is geometrically complex and dominated by large areas of thin wall sections with cut-outs, thin walls orthogonal structures and thickness variations. The flow is complex and involves fragmented jets flowing rapidly to the perimeter of the die and then back filling into the voids that remained behind. The last areas to fill, where residual air is likely to be trapped and produce porosity, are around the gates where the high speed jets fragment and spray across towards the back filling fluid surface. Die filling completes when this back filling surface reaches all the gates.

The predicted filling patterns have been compared to water analogue experiments (with matched Reynolds numbers) and found to be in very good agreement with key flow structures matching in all four die quadrants. In particular:

- The relative rate of movement of the flow fronts from each of the gates,

- The timing and volume of fluid reaching and accumulating on obstacles and cut-outs,

- The shapes and locations of the voids left between the jets from the gates during early filling, and

- The location and relative rate of where the back filling process begins.

A few local differences in flow were observed, most particularly the presence of the thick slower moving band of fluid at the start of the injection process, which will require further investigation.

Several of the flow features predicted are visually identifiable in the real cast parts (after etching). In particular, there are strong matches between the flows lines for the flow from the gates to the vents that occur after filling is complete and which are frozen into the final casting. Key predicted recirculation structures predicted by the SPH model also matched with visually similar frozen structures in the casting.

The combination of the matching water analogue experiments and good correlation of the predicted flow to final casting microstructure demonstrates the suitability of SPH as a computational tool for predicting complex thin wall die filling behaviour. As economic pressure and the requirement for ever lighter component weights (particularly in automotive and personal electronic devices) increases, it is important that the numerical tools that can be used to assist with casting system design be demonstrated to be sufficiently accurate for these increasingly thin components. This study provides a useful degree of confidence that the prediction accuracy found in earlier studies of bulkier components extends to these newer and thinner parts.

The predicted flow (both SPH and water analogue) indicates that there are issues for this particular die, such as balancing mass flow rates through the various gates, the shape of the fan gate, the positioning of the shot sleeve and the amount and location of the venting that can be optimized for faster filling and more even flow.

Acknowledgments This work was performed in conjunction with the 2000 NIST/ATP \#70NANBOH3053 — Cost Reduced Magnesium Die Castings Using Heated Runners.

\section{References}

1. Bonet J, Kulasegaram S (2000) Correction and stabilization of smooth particle hydrodynamics methods with applications in metal forming simulations. Int J Numer Methods Eng 47:1189-1214

2. Cleary PW (2010) Extension of SPH to predict feeding, freezing and defect creation in low pressure die casting. Appl Math Model 34(11):3189-3201

3. Cleary PW, Ha J, Ahuja V (2000) High pressure die casting simulation using Smoothed Particle Hydrodynamics. J Cast Metals Res 12:335-355

4. Cleary PW, Ha J (2000) Three dimensional modelling of high pressure die casting. Int J Cast Metals Res 12:357-365

5. Cleary PW, Ha J (2002) Flow modelling in casting processes. Appl Math Model 26:171-190

6. Cleary PW, Prakash M, Ha J, Sinnott M, Nguyen T, Grandfield J (2004) Modelling of cast systems using smoothed particle hydrodynamics. J Met 55:67-70

7. Cleary PW, Ha J, Prakash M, Nguyen T (2006) 3D SPH flow predictions and validation for high pressure die casting of automotive components. Appl Math Model 30:1406-1427

8. Cleary PW, Prakash M, Ha J (2006) Novel applications of SPH in metal forming. J Mater Proces Technol 177:41-48 
9. Cleary PW, Prakash M, Ha J, Stokes N, Scott C (2007) Smooth particle hydrodynamics: status and future potential. Prog Comput Fluid Dyn 7:70-90

10. Cleary PW, Ha J, Prakash M (2012) Modelling of metal forging using SPH. Appl Math Model 36:3836-3855

11. Cleary PW, Ha J, Prakash M, Nguyen T (2010) Short shots and industrial cases studies: understanding fluid flow and solidification in high pressure die casting. Appl Math Model 34:2018-2033

12. Davis AJ, Asquith SJ (1985) Water analogue studies of fluid flow in the die casting process. In: SDCE 13th international die casting congress and exposition, Milwaukee, Wisconsin, 3-6 June 1985 (G-T85-063), p. 6

13. Gunasegaram DR, Farnsworth DJ, Nguyen TT (2009) Identification of critical factors affecting shrinkage porosity in permanent mold casting using numerical simulations based on design of experiments. J Mater Process Technol 209:1209-1219

14. Ha J, Cleary PW (2000) Comparison of SPH simulations of high pressure die casting with the experiments and VOF simulations of Schmid and Klein. Int J Cast Metals Res 12:409-418

15. Ha J, Schuhmann R, Alguine V, Cleary P, Nguyen T (2000) Realtime Xray imaging and numerical simulation of die filling in gravity die casting. In: Proceedings of the modeling of casting, welding and advanced solidification processes IX, pp 151-158

16. Ha J, Cleary PW (2005) Simulation of high pressure die filling of a moderately complex industrial object using smoothed particle hydrodynamics. Int J Cast Met Res 18:81-92

17. Helenius R, Lohne O, Arnberg L, Laukli HI (2005) The heat transfer during filling of a high-pressure die-casting shot sleeve. Mater Sci Eng A 413-414:52-55

18. Hu BH, Tong KK, Niu XP, Pinwill I (2000) Design and optimisation of runner and gating systems for the die casting of thin-walled magnesium telecommunication parts through numerical simulation. J Mater Process Technol 105:128-133

19. Kermanpur A, Mahmoudi Sh, Hajipour A (2008) Numerical simulation of metal flow and solidification in the multi-cavity casting moulds of automotive components. J Mater Process Technol 206:62-68

20. Lee YS, Lee SU, Van Tyne CJ, Joo BD, Moon YH (2011) Internal void closure during the forging of large cast ingots using a simulation approach. J Mater ProcessTechnol 211(6):1136-1145

21. Monaghan JJ (1992) Smoothed particle hydrodynamics. Annu Rev Astron Astrophys 30:543-574

22. Monaghan JJ (1994) Simulating free surface flows with SPH. J Comput Phys 110:399-406
23. Monaghan JJ (2005) Smoothed particle hydrodynamics. Rep Prog Phys 68(8):1703-1759

24. Pang S, Chen L, Zhang M, Yin Y, Chen T, Zhou J, Liao D (2010) Numerical simulation two phase flows of casting filling process using SOLA particle level set method. Appl Math Model 34(12):4106-4122

25. Pellika EA, Wendt J (2003) How high-pressure die-cast component (HPDC) foundries benefit from numerical simulation. Adv Eng Mater 5(1-2):58-61

26. Pineau F, D'Amours G (2012) SPH model approach used to predict skin inclusions into semi-solid metal castings. In: Nastac L et al (eds) CFD modelling and processing in materials processing. Wiley, Hoboken (Chap. 26)

27. Prakash M, Cleary PW, Grandfield J, Rohan P, Nguyen V (2007) Optimisation of ingot casting wheel design using SPH simulations. Prog Comput Fluid Dyn 7(2-4):101-110

28. Prakash M, Cleary P, Grandfield J (2009) Modelling of metal flow and oxidation during furnace emptying using smoothed particle hydrodynamics. J Mater Process Technol 209:3396-3407

29. Prakash M, Pereira GG, Cleary PW, Rohan P, Taylor JA (2010) Validation of SPH predictions of oxide generated during Al melt transfer. Prog Comput Fluid Dyn 10:319-326

30. Prakash M, Cleary PW, Taylor J, Harker B (2011) SPH modeling of the effect of crucible tipping rate on oxide formation. Mater Sci Forum 693:54-62

31. Rai JK, Lajimi AM, Xirouchakis P (2008) An intelligent system for predicting HPDC process variables in interactive environment. J Mater ProcessTechnol 203(1-3):72-79

32. Rider WJ, Kothe DB (1998) Reconstructing volume tracking. J Comput Phys 141:112-152

33. Rudman M (1997) Volume-tracking methods for interfacial flow calculations. Int J Numer Methods Fluids 24:671-691

34. Schneider W Grun GU (1995) The new airsol veil billet casting system-improvements, mathematical modelling, cast house experiences. In: Fourth Australian Asian Pacific aluminium cast house technology conference (TMS), pp 296-315.

35. Vijaykumar A (2012) Smoothed particle hydrodynamics simulation for continuous casting. Masters Thesis in Scientific Computing, KTH, Stockholm 